\section{PROMOÇÃO DA ALIMENTAÇÃO SAUDÁVEL ENTRE PROFISSIONAIS DE SAÚDE NA ATENÇÃO PRIMÁRIA}

\author{
Promotion of healthy eating among health professionals in \\ primary care
}

\author{
Promoción de alimentación saludable entre profesionales \\ sanitarios de la atención primaria
}

\title{
RESUMO
}

Objetivo: Relatar a experiência da implantação de ações educativas sobre alimentação saudável entre profissionais de saúde na Estratégia Saúde da Família. Síntese dos dados: Foram aplicadas capacitações in loco em 50 equipes de Estratégia Saúde da Família, no período de dezembro de 2014 a março de 2015 . Houve a participação de profissionais de saúde, como médicos, enfermeiros, técnicos de enfermagem, dentistas e agentes comunitários de saúde, participantes do projeto das Unidades Promotoras da Saúde, na cidade de Montes Claros, MG. Foram realizadas 4 oficinas, com duração média de 50 minutos cada. Nas oficinas, foram desenvolvidas atividades dinâmicas sobre hábitos alimentares saudáveis, avaliação antropométrica, índice de massa corporal, consumo alimentar, análise de rótulos dos alimentos e discussão sobre as atividades exercidas visando à motivação e atenção voltada à promoção da saúde da população, incentivando a educação alimentar e nutricional para a formação de hábitos alimentares saudáveis. Conclusão: A experiência de implantação de ações educativas sobre alimentação saudável possibilitou a construção de novos conceitos sobre o tema entre os profissionais de saúde na Estratégia Saúde da Família. Dessa forma, as oficinas em alimentação saudável podem ser uma ferramenta para mudança da realidade de práticas em alimentação e nutrição desses profissionais no seu território de atuação. A capacitação dos profissionais de saúde beneficiará a população ao potencializar as ações de promoção de saúde e de conscientização para prevenção dos agravos à saúde relacionados à má alimentação na atenção básica.

Descritores: Alimentação; Promoção da Saúde; Hábitos Alimentares.

\section{ABSTRACT}

Objective: To report the experience of implementing educational activities regarding healthy eating among health professionals in the Family Health Strategy. Data synthesis: In loco training was provided for 50 Family Health Strategy teams in the period from December 2014 to March 2015. There was the participation of health professionals such as doctors, nurses, nursing technicians, dentists and community health workers who were participants in the project of Health Promoting Units, in the city of Montes Claros, MG. Four workshops were held, with average duration of 50 minutes each. In the workshops, dynamic activities were developed covering healthy eating habits, anthropometric measurements, body mass index, food consumption, analysis of food labels, and discussion about the activities performed aimed at motivation and attention towards the population's health promotion, stimulating food and nutrition education for the development of healthy eating habits. Conclusion: The experience of implementing educational actions on healthy eating made it possible to construct new concepts on the subject among health professionals in the Family Health Strategy. Thus, the workshops on healthy eating can be a tool to change the reality of practices in food and nutrition of these professionals in their territory of action. Training the health professionals will benefit the population by strengthening the actions of health promotion and awareness for prevention of health conditions related to poor diet in primary health care.

1) Faculdades Unidas do Norte de Minas - FUNORTE/SOEBRAS - Montes Claros (MG) - Brasil

Descriptors: Feeding; Health Promotion; Food Habits.

Recebido em: 14/09/2016 Revisado em: 18/10/2016 Aceito em: 29/11/2016 


\section{RESUMEN}

Objetivo: Relatar la experiencia de la implantación de acciones educativas sobre la alimentación saludable entre profesionales sanitarios de la Estrategia Salud de la Familia. Síntesis de los datos: Se realizaron formaciones in loco en 50 equipos de Estrategia Salud de la Familia entre diciembre de 2014 y marzo de 2015. Hubo la participación de profesionales sanitarios como médicos, enfermeros, técnicos de enfermería, dentistas y agentes comunitarios de salud que eran participantes del proyecto de las Unidades Promotoras de Salud en la ciudad de Montes Claros, $M G$. Se realizaron 4 talleres con una media de duración de 50 minutos cada uno. En los talleres se desarrollaron actividades dinámicas sobre los hábitos alimentarios saludables, la evaluación antropométrica, el índice de masa corporal, el consumo alimentario, el análisis de los rótulos de los alimentos y discusión sobre las actividades ejecutadas objetivando la motivación y la atención direccionada a la promoción de la salud de la población con incentivo a la educación alimentaria y nutricional para la formación de hábitos alimentarios saludables. Conclusión: La experiencia de implantación de acciones educativas sobre la alimentación saludable posibilitó la construcción de nuevos conceptos sobre el tema entre los profesionales sanitarios de la Estrategia Salud de la Familia. Así, los talleres de alimentación saludable pueden ser una herramienta para cambios en las prácticas de alimentación y nutrición de esos profesionales en su territorio de actuación. La formación de los profesionales sanitarios ofrecerá beneficios a la población por potenciar las acciones de promoción de salud y de concienciación para la prevención de los agravios a la salud relacionados a la mala alimentación de la atención básica.

Descriptores: Alimentación; Promoción de la Salud; Hábitos Alimenticios.

\section{INTRODUÇÃO}

A Estratégia Saúde da Família (ESF) é definida como porta de entrada do sistema de saúde no Brasil ${ }^{(1)}$. Além disso, ela adapta mudanças positivas que beneficiam a vida da população, realizando, assim, ações com profissionalismo e responsabilidade para a construção de aspectos necessários para a melhoria da saúde da população, atribuindo uma visão diferenciada de cuidado nutricional ao longo da vida, como conhecimento e prazer no consumo de alimentos saudáveis ${ }^{(1,2)}$.

A promoção da alimentação saudável é primordial para a conscientização dos profissionais de saúde, uma vez que é direcionada para o fortalecimento preventivo de patologias, tendo como foco efetivar a promoção da saúde e abrangendo todas as classes, independente de idade, sexo, etnia, etc. Trata-se de uma ferramenta potencial para que se obtenha uma estrutura de vida saudável ${ }^{(3)}$.
As ações de alimentação e nutrição na atenção básica podem contribuir tanto para a qualificação como para a garantia da integralidade da atenção à saúde prestada à população brasileira ${ }^{(4)}$. A partir dessa premissa, faz-se importante o incentivo aos profissionais da ESF para o trabalho com temáticas dessa natureza em sua atuação.

O profissional de saúde, durante a sua atuação nas ações de educação em saúde, deve ser inovador, promovendo ações educativas que busquem fazer com que o sujeito reflita e transforme suas práticas anteriores, de modo que entenda que a incorporação dessas ações ao seu cotidiano poderá beneficiar ainda mais a sua saúde, favorecendo a prevenção de doenças e promovendo hábitos de vida saudáveis ${ }^{(5)}$.

Entretanto, deve-se levar em consideração o meio em que o individuo está inserido, pois, nessa interação, haverá uma consciência mútua que resultará em crescimento e autonomia $^{(6)}$. Com a conscientização por parte do profissional de saúde e do paciente, acontecerá uma mudança no seu estilo de vida, resultando em qualidade e prevenção de patologias ${ }^{(5)}$.

Nesse sentido, torna-se necessária a utilização de estratégias para promover uma aprendizagem efetiva, embasada no pensamento reflexivo, dialógico, contextual, colaborativo e construtivo ${ }^{(7,8)}$. Além disso, é importante ressaltar que o interesse, comprometimento e esforço dos profissionais de saúde na aquisição de conhecimentos contribuem para a transformação de sua prática profissional e consequentemente impactam no seu atendimento ${ }^{(9)}$.

A alimentação adequada vem sendo introduzida de forma gradativa nas ações de promoção da saúde, baseada principalmente na conscientização do indivíduo. $O$ profissional nutricionista atua com o intuito de promover hábitos alimentares no desenvolvimento de um estilo de vida saudável na população, de modo a contribuir na garantia da qualidade de vida ${ }^{(10)}$.

$\mathrm{Na}$ atenção primária, a orientação para alimentação saudável ainda é um desafio e, por isso, a necessidade de maiores esforços no sentido de ampliar a oferta ${ }^{(4,11)}$. Portanto, no presente trabalho, objetivou-se relatar a experiência da implantação de ações educativas sobre alimentação saudável entre profissionais de saúde na Estratégia Saúde da Família.

\section{SÍNTESE DOS DADOS}

Trata-se de um relato de experiência, realizado em 50 equipes de Estratégia Saúde da Família (ESF) integrantes do projeto das Unidades Promotoras de Saúde, na cidade de Montes Claros - MG, no período de dezembro de 2014 a março de 2015. Participaram os profissionais de saúde cadastrados na ESF, tais como médicos, enfermeiros, 
técnicos de enfermagem, dentistas e agentes comunitários de saúde, que aceitaram o convite. Uma vez que os profissionais da ESF são o elo do sistema de saúde com a população, o trabalho foi realizado através de oficinas com rodas de discussão, debates, dinâmicas e realização de atividades práticas com objetivo de aplicar ações educativas sobre alimentação saudável entre profissionais de saúde, acarretando verificação direcionada à educação alimentar e nutricional para a formação de bons hábitos alimentares.

A atividade educativa consistiu em quatro oficinas, com frequência semanal e duração de 50 minutos cada, conduzidas por profissionais de nutrição devidamente treinados. As oficinas aconteceram na ESF, com a participação voluntária de aproximadamente 15 profissionais por equipe. O número dos participantes variou em cada equipe de ESF considerando a disponibilidade. Nas oficinas, eram abordados temas referentes à alimentação, nutrição e saúde, em um espaço que propiciava a socialização e troca de experiência entre os participantes. Estimulou-se o pensamento crítico na construção do conhecimento, de modo a garantir o empoderamento do sujeito. No quadro I consta a descrição das oficinas.

Quadro I - Descrição das Oficinas realizadas com os profissionais de saúde. Montes Claros, MG, 2014-2015.

\begin{tabular}{|c|c|c|c|c|}
\hline Oficinas & Tema & Objetivo & Conteúdo & Encaminhamentos \\
\hline Oficina 01 & Hábito alimentar & $\begin{array}{l}\text { Discutir os hábitos } \\
\text { alimentares da } \\
\text { população }\end{array}$ & $\begin{array}{l}\text { Guia alimentar } \\
\text { para a população } \\
\text { brasileira }\end{array}$ & $\begin{array}{l}\text { Reflexão coletiva dos participantes } \\
\text { sobre a importância da adoção de } \\
\text { hábitos alimentares saudáveis. }\end{array}$ \\
\hline Oficina 02 & $\begin{array}{c}\text { Alimentação e } \\
\text { Saúde }\end{array}$ & $\begin{array}{c}\text { Avaliar o estado } \\
\text { nutricional e a relação } \\
\text { com o consumo } \\
\text { alimentar }\end{array}$ & $\begin{array}{l}\text { Avaliação } \\
\text { Nutricional }\end{array}$ & $\begin{array}{l}\text { Reflexão coletiva dos participantes } \\
\text { sobre o impacto do consumo } \\
\text { alimentar sobre a saúde. }\end{array}$ \\
\hline Oficina 03 & $\begin{array}{l}\text { O que estou } \\
\text { comendo? }\end{array}$ & $\begin{array}{c}\text { Analisar os rótulos de } \\
\text { alimentos }\end{array}$ & $\begin{array}{l}\text { Rotulagem } \\
\text { Nutricional }\end{array}$ & $\begin{array}{c}\text { Reflexão coletiva dos participantes } \\
\text { sobre a qualidade dos alimentos } \\
\text { consumidos. }\end{array}$ \\
\hline Oficina 04 & $\begin{array}{l}\text { Planejamento } \\
\text { das ações de } \\
\text { alimentação e } \\
\text { compromisso com } \\
\text { a mudança }\end{array}$ & $\begin{array}{l}\text { Implementar a prática } \\
\text { de uma alimentação } \\
\text { saudável }\end{array}$ & Motivacional & $\begin{array}{c}\text { Reflexão coletiva dos participantes } \\
\text { sobre as estratégias cotidianas para } \\
\text { o consumo de uma alimentação } \\
\text { saudável. }\end{array}$ \\
\hline
\end{tabular}

\section{Oficina 1 - Hábito alimentar}

$\mathrm{Na}$ primeira oficina, discutiu-se com os participantes sobre os seus hábitos alimentares diários. A dieta habitual dos brasileiros é influenciada por diversos fatores, e, atualmente, é caracterizada com padrão de consumo "tradicional" (baseada no arroz com feijão) associado aos alimentos classificados como ultraprocessados, com altos teores de gorduras, sódio, açúcar e calorias e um baixo teor de micronutrientes. $\mathrm{O}$ consumo médio de frutas e hortaliças ainda não atende as recomendações do Guia Alimentar para a população brasileira, enquanto alimentos ultraprocessados, como doces e refrigerantes, têm o seu consumo aumentado a cada ano ${ }^{(12)}$.

A dinâmica realizada na Oficina 1, baseada no Guia Alimentar para a População Brasileira, avaliou a percepção dos participantes sobre como seria uma refeição considerada saudável. Cada participante desenhou a sua refeição habitual na figura de um prato.
Após a discussão e avaliação dos hábitos alimentares dos participantes, distribuiu-se o folder, elaborado pelo Ministério da Saúde, "10 passos para uma alimentação saudável". Esse material orientou a nova discussão no grupo sobre alimentação saudável e maneiras de manter/ alcançar uma melhor qualidade de $\operatorname{vida}^{(13)}$.

A alimentação saudável e hábitos alimentares adequados são fatores indispensáveis na proteção contra doenças crônicas e no alcance e manutenção de uma boa qualidade de vida. Portanto, a mudança dos hábitos alimentares é imprescindível para a saúde e o bem-estar do individuo $^{(14)}$.

Na promoção da alimentação saudável nos espaços de atuação, o profissional precisa ter uma base teórica sobre esse conteúdo. Em estudo realizado entre profissionais da educação, que são parceiros da atenção básica no mesmo município, observou-se uma limitação de conhecimento sobre o tema, e ainda relataram carecer de uma capacitação 
específica sobre conteúdos em Alimentação Saudável ${ }^{(15)}$. Nesse sentido, é importante que, entre o contingente de profissionais que atuam na atenção básica, sejam realizadas iniciativas como a apresentada no estudo, para que possam adquirir os conhecimentos específicos e garantir o sucesso nas ações de promoção da alimentação saudável no território.

\section{Oficina 2 - Alimentação e saúde}

A alimentação é muito mais do que nutrição uma vez que os alimentos são preparados e combinados entre si. Além disso, o ato de alimentar-se é influenciado por hábitos e costumes do indivíduo, o que gera influência direta na sua saúde e bem-estar. Com base nessa premissa, a segunda oficina destacou a importância da alimentação saudável relacionando o consumo alimentar diário e o estado nutricional $^{(14)}$.

Estima-se que $70 \%$ das mortes na população adulta, no Brasil, ocorram devido às doenças crônicas não transmissíveis. É sabido, porém, que a alimentação possui grande influência no desenvolvimento ou não dessas condições ${ }^{(1)}$.

Para relacionar a alimentação e a saúde, analisaram-se os hábitos alimentares dos participantes (ingestão de frutas e verduras, consumo hídrico e intervalo entre as refeições).

Em seguida, a partir da avaliação antropométrica individual, procedeu-se a orientação nutricional com base na sua condição, riscos iminentes, quando aplicáveis, e medidas de prevenção de patologias associadas.

\section{Oficina 3 - O que estou consumindo?}

A Oficina 3 consistiu em uma revisão dos encontros anteriores e uma reflexão sobre a mudança alimentar dos participantes ao longo das intervenções. Além disso, nessa oficina, os participantes debateram sobre os rótulos de alimentos, atualmente considerados ferramenta de comunicação entre as empresas que produzem os alimentos e os consumidores, com a finalidade de informá-los sobre os alimentos adquiridos.

Regulamentações sobre rotulação alimentar são vistas como importantes atividades de promoção da saúde. O consumidor tem direito ao acesso às informações sobre as características e composição nutricional dos alimentos que adquire no comércio, ou seja, às suas propriedades nutricionais, para satisfazer suas necessidades alimentares. A rotulagem dos alimentos, ao orientar o consumidor sobre a qualidade e a quantidade dos constituintes nutricionais dos produtos, pode promover escolhas alimentares apropriadas, sendo indispensável, no entanto, a implementação continuada de intervenções no âmbito da promoção da saúde ${ }^{(16)}$.
Assim, nessa oficina, procedeu-se a avaliação de rótulos de alimentos, previamente solicitados aos participantes, a fim de detectar a presença de substâncias causadoras de patologias associadas ao consumo excessivo. Com base nos rótulos, esclareceram-se as dúvidas relacionadas à nomenclatura, às substâncias e às quantidades encontradas nos rótulos de alimentos, finalizando com uma discussão com intenção preventiva sobre deslizes e recaídas durante a tentativa de implantação de dietas saudáveis.

\section{Oficina 4 - Planejamento das ações de alimentação e compromisso com a mudança}

A Oficina 4 teve como objetivo principal recapitular todas as atividades realizadas anteriormente e implementar estratégias no cotidiano para a adoção de hábitos alimentares saudáveis. Além disso, ressaltar a importância da motivação para a realização de mudanças na alimentação. Os participantes assistiram a um vídeo educativo, com o intuito de motivar a prática de hábitos alimentares saudáveis.

A educação nutricional é uma das ações indispensáveis nos cuidados relativos à alimentação e à nutrição na Atenção Básica, e envolve a promoção de práticas alimentares adequadas e saudáveis. Essas ações devem priorizar o desenvolvimento de habilidades e competências que gerem empoderamento e autonomia nos indivíduos, famílias e comunidades, considerando abordagens que levem em conta as especificidades do ciclo da vida ${ }^{(17)}$.

A avaliação do aprendizado foi realizada individualmente, a partir de questionamentos sobre todas as atividades realizadas, e cada profissional teve o seu momento para esclarecer possíveis dúvidas.

Como atividade de encerramento da oficina, era oferecido um lanche coletivo, em que cada participante compartilhava um alimento saudável de sua preferência. O objetivo desse lanche foi propiciar a confraternização e demonstrar a factibilidade da prática da alimentação saudável no seu contexto social.

$\mathrm{Na}$ atenção primária à saúde, a orientação para alimentação saudável não é universal e há iniquidade, deixando clara a necessidade de maiores esforços no sentido de ampliar a oferta. A promoção de práticas alimentares adequadas e saudáveis, recomendada pelas políticas e programas de saúde, ainda não atingi efetivamente o usuário do sistema na Atenção Primária à Saúde ${ }^{(18)}$.

Nesse sentido, a intervenção nutricional, com base na realização de oficinas, pode ser considerada uma ferramenta potencial entre profissionais de saúde para aquisição de informações em nutrição e mudança de hábitos alimentares. As ações educativas impactam em uma prática consciente de comportamentos para promoção da saúde e favorecem uma vida saudável ${ }^{(19)}$. 
Além disso, a implantação de oficinas em uma perspectiva de educação permanente tem sido evidenciada como espaço para reflexão, integração e construção de ideias para a prática. Os espaços de Educação Permanente devem ser construídos com os profissionais para apoiar e fomentar as ações de promoção da alimentação saudável no território de ação ${ }^{(20)}$.

Esse estudo respeitou os preceitos éticos, sendo aprovado pelo Comitê de Ética em Pesquisa da instituição sede da pesquisa sob o parecer $n^{\circ}$. 1.145.637/2015.

\section{CONCLUSÃO}

A experiência de implantação de ações educativas sobre alimentação saudável possibilitou a construção de novos conceitos sobre o tema entre os profissionais de saúde na Estratégia Saúde da Família. Dessa forma, as oficinas em alimentação saudável podem ser uma ferramenta para mudança na realidade de práticas em alimentação e nutrição desses profissionais no seu território de atuação. A capacitação dos profissionais de saúde beneficiará a população ao potencializar as ações de promoção de saúde e de conscientização para prevenção dos agravos à saúde relacionados à má alimentação na atenção básica.

\section{REFERÊNCIAS}

1. Ministério da Saúde (BR). Portaria $n^{\circ}$. 2.488, de 21 de outubro de 2011. Aprova a Política Nacional da Atenção Básica, estabelecendo a revisão de diretrizes e normas para a organização da Atenção Básica, para a Estratégia Saúde da Família (ESF) e o Programa de Agentes Comunitários de Saúde (PACS). Brasília: Ministério da Saúde; 2011.

2. Recine E, Vasconcelos AB. Políticas nacionais e o campo da Alimentação e Nutrição em Saúde Coletiva: cenário atual. Ciênc Saúde Coletiva. 2011;16(1):73-9.

3. Yokota RTC, Vasconcelos TF, Pinheiro ARO, Schmitz BAbS, Coitinho DC, Rodrigues MLCF. Projeto "A escola promovendo hábitos alimentares saudáveis": comparação de duas estratégias de educação nutricional no Distrito Federal, Brasil. Rev Nutr. 2010;23(1):3747.

4. Pimentel VRM, Sousa MF, Hamann EM, Mendonça AVM. Alimentação e nutrição na Estratégia Saúde da Família em cinco municípios brasileiros. Ciênc Saúde Coletiva. 2014;19(1):49-58.

5. Moreira AC. Unidades promotoras de saúde. Manual de implantação. Belo Horizonte: Secretaria do Estado de Saúde de Minas Gerais; 2014.
6. Silva KL, Sena RR. Poder, autonomia e responsabilização: promoção da saúde em espaços sociais da vida cotidiana. São Paulo: Hucitec; 2010.

7. Guimarães EMP, Martin SH, Rabelo FCP. Educação Permanente em Saúde: Reflexão e Desafios. Cienc Enferm. 2010;16(2):25-33.

8. Falkenberg MB, Mendes TPL, Moraes EP, Souza EM. Educação em saúde e educação na saúde: conceitos e implicações para a saúde coletiva. Ciênc Saúde Coletiva. 2014;19(3):847-52.

9. Rodrigues ACS, Vieira GLC, Torres HC. A proposta da educação permanente em saúde na atualização da equipe de saúde em diabetes mellitus. Rev Esc Enferm USP. 2010;44(2):531-7.

10. Ferreira VA, Magalhães R. Nutrição e promoção da saúde: perspectivas atuais. Cad Saúde Pública. 2007;23(7):1674-81.

11. Soares DG, Pinheiro MCX, Queiroz DM, Soares DG. Implantação da puericultura e desafios do cuidado na estratégia saúde da família em um município do Estado do Ceará. Rev Bras Promoç Saúde. 2016;29(1):132-8.

12. Ministério da Saúde (BR). Política Nacional de Alimentação e Nutrição. Brasília: Ministério da Saúde; 2012.

13. Ministério da Saúde (BR). Guia alimentar para a população brasileira. Brasília: Ministério da Saúde; 2014.

14. World Health Organization, Food and Agriculture Organization. Diet, nutrition and the prevention of chronic diseases: report of a joint $\mathrm{WHO} / \mathrm{FAO}$ expert consultation. Geneva; 2002.

15. Bezerra KF, Capuchinho LCFM, Pinho L. Conhecimento e abordagem sobre alimentação saudável por professores do ensino fundamental. DEMETRA. 2015;10:119-31.

16. Souza SMFC, Lima KC, Alves MSCF. A rotulagem nutricional para escolhas alimentares mais saudáveis: estudo de intervenção, Natal - RN. Vigil Sanit Debate. 2014;2(1):64-8.

17. Jaime PC, Santos LMP. Transição nutricional e a organização do cuidado em alimentação e nutrição na Atenção Básica em saúde. Divulg Saúde Debate. 2014;51:72-85.

18. Lindemann IL, Mendoza-Sassi RA. Orientação para alimentação saudável e fatores associados entre usuários da atenção primária à saúde no sul do Brasil. Rev Bras Promoç Saúde. 2016;29(1):34-42. 
19. Silva CP, Carmo AS, Horta PM, Santos LC. Intervenção nutricional pautada na estratégia de oficinas em um serviço de promoção da saúde de Belo Horizonte, Minas Gerais. Rev Nutrição. 2013;26(6):647-58.

20. Juzwiak CR, Castro PM, Batista SHSS. A experiência da Oficina Permanente de Educação Alimentar e em Saúde (OPEAS): formação de profissionais para a promoção da alimentação saudável nas escolas. Ciênc Saúde Coletiva. 2013;18(4):1009-18.
Endereço do primeiro autor: Sarah Fernanda Barbosa Frois

Faculdades Unidas do Norte de Minas - FUNORTE/ SOEBRAS

Avenida Osmane Barbosa, 1111

Bairro JK

CEP 39404-006 - Montes Claros - MG - Brasil

E-mail: nanda.frois@hotmail.com

Endereço para correspondência:

Lucineia de Pinho

Faculdades Unidas do Norte de Minas - FUNORTE/

SOEBRAS

Avenida Osmane Barbosa, 1111,

Bairro JK

CEP 39404-006 - Montes Claros - MG - Brasil

E-mail: lucineiapinho@hotmail.com 